\title{
Contact-induced spin polarization in carbon nanotubes
}

\author{
Mauro S. Ferreira and Stefano Sanvito \\ Physics Department, Trinity College, Dublin 2, Ireland \\ (Received 27 June 2003; published 16 January 2004)
}

\begin{abstract}
Motivated by the possibility of combining spintronics with molecular structures, we investigate the conditions for the appearance of spin polarization in low-dimensional tubular systems by contacting them to a magnetic substrate. We derive a set of general expressions describing the charge transfer between the tube and the substrate and the relative energy costs. The mean-field solution of the general expressions provides an insightful formula for the induced spin polarization. Using a tight-binding model for the electronic structure we are able to estimate the magnitude and the stability of the induced moment. This indicates that a significant magnetic moment in carbon nanotubes can be observed.
\end{abstract}

DOI: 10.1103/PhysRevB.69.035407

PACS number(s): 61.46.+w, 68.65.La

\section{INTRODUCTION}

Over the last decade there has been an explosive increase of activity in two key areas of material science: spintronics and molecular electronics. Spintronics is based on the use of the spin degrees of freedom as well as the electronic charge for a number of applications. ${ }^{1}$ The field has expanded significantly since the discovery of the giant magnetoresistance effect in magnetic multilayers ${ }^{2}$ and has been the main driving force leading to the development of the present generation of magnetic storage devices.

Although the field has already demonstrated part of its potential, it is worth noting that most of the proposed applications simply translate well known concepts of conventional electronics into spin systems. The typical devices are made with molecular-beam-epitaxy growth, and lithographic techniques; a bottom-up approach to spintronics devices has scarcely been explored. In this respect molecular electronics provides the opposite approach. ${ }^{3}$ Here the basic idea is to use molecular systems for electronic applications and conventional electronic devices such as transistors,${ }^{4}$ negative differential resistors, ${ }^{5}$ and rectifiers ${ }^{6}$ have already been produced at the molecular level.

A few experiments have attempted to combine spintronics with molecular devices. In their pioneering experiment ${ }^{7}$ Tsukagoshi and co-workers demonstrated that the $I-V$ curve of a carbon nanotube sandwiched between two Co contacts presents hysteresis when a magnetic field is applied. Such spin-valve behavior indicates spin injection into the nanotube with a spin diffusion length (the average distance that an electron travels before flipping its spin direction) of the order of $100 \mathrm{~nm}$. This makes carbon nanotubes very attractive for spintronics applications. Other carbon structures are capable of accommodating net spin polarization and Coey et al. have shown evidence for a strong induced magnetic polarization at room temperature in a graphite system with embedded ferromagnetic nanoclusters. ${ }^{8}$

From these experiments it emerges that, on the one hand spins can propagate in carbon materials almost without flipping their direction, and on the other that the proximity with magnetic materials can induce spin polarization in graphitebased systems. Although more controlled experiments on synthetic nanostructures are needed, we believe that the implementation of spin physics in carbon systems is possible and it will be crucial for the development of smaller and more sophisticated magnetotransport devices.

Motivated by the idea of combining spintronics with molecular structures, we investigate the conditions for which an induced spin polarization appears in a low-dimensional tubular molecule contacted to a magnetic material. Although carbon nanotubes are the immediate motivation for this work, our formalism is rather general and can be applied to any cylindrical structure.

This paper is organized as follows. In the following section, we derive a general expression for the charge transfer that occurs when a tubular molecule is side contacted to a metallic substrate. A complementary expression for the contact-induced total energy change is also presented. This set of expressions determines not only how the charge is redistributed when the tubular molecule is contacted to the substrate but also provides information about the stability of the transfer process. The expressions derived in Sec. II do not present any explicit spin dependence. In Sec. III we generalize them to include the spin asymmetry of magnetic substrates and we demonstrate that a net magnetic moment can be induced in the tubular molecule. An estimation of the magnitude of the induced magnetic moment and of its stability is then given.

\section{CONTACT-INDUCED CHARGE TRANSFER AND ENERGY GAIN}

In order to investigate how a magnetic contact affects the spin polarization of a nanotube, we start by calculating the change in the electronic structure of a tubular molecule side contacted to a substrate. We model the contact by introducing an electronic coupling between the tube and the substrate that accounts for the possible charge transfer between the two materials. The interaction is assumed to be only between the two lines of atoms, one on the tube and one on the substrate, that are in closest proximity. Spin-dependent charge transfer is likely to arise due to the spin asymmetry of the magnetic substrate, thus leading to a net induced spin polarization.

The spin-dependent density of states is the relevant quantity to calculate and provides the necessary information 
about whether or not the contact leads to a net polarization of the nanostructure. We start by making no assumptions regarding the specific models describing the electronic structure of the system. In this way, we express the spin-polarized density of states in terms of single-particle Green functions matrix elements that can be calculated by different techniques based on model Hamiltonians. Such a modelindependent treatment emphasizes the generality of our results and leads to a set of closed-form expressions that provide a general method to investigate charge transfer between contacting materials.

We consider infinitely long tubes of diameter $d$, which can be thought of as two-dimensional finite-width stripes wrapped around in cylindrical shape. We also assume that $N$ atoms are placed along the circumference. The tubular system shows translational symmetry along the longitudinal direction. Therefore, the electronic states along this direction are well described by a reciprocal-space wave vector that runs within the one-dimensional Brillouin zone. Since the translational symmetry is broken by the line of atoms contacting the substrate, it is convenient to use real space coordinates along the circumferential direction. In this way, electronic states are identified by the pair of indices $(j, k)$, where $k$ corresponds to the wave vector along the longitudinal direction and $j$ labels the $N$ lines of atoms on the tube surface. Since the substrate also has translational symmetry along the axial direction of the tube, the electronic states of the substrate can be labeled by the same pair of indices.

In terms of the single-particle Green function, the total density of states $\rho(E)$ is written as

$$
\rho(E)=\left(-\frac{1}{\pi}\right) \operatorname{Im} \sum_{k} \sum_{j} G_{j, j}(E, k),
$$

where $G_{j, j}(E, k)$ is the Green function of an electron with energy $E$ moving on line $j$ with wave vector $k$. The sum over $j$ accounts for all the atomic lines of the tube and the substrate.

It is convenient to define another Green function associated with the disconnected system, that is, the isolated substrate and tube. In this case, the translational symmetry along the circumferential direction is reestablished and the electronic states on the tube are usually described by a set of two wave vectors; the longitudinal component running continuously over the one-dimensional Brillouin zone and a finite set of quantized wave vectors induced by the cylindrical boundary conditions. Likewise, the in-plane translational symmetry is also restored for the substrate. However, to be consistent with the notation in terms of the indices $(j, k)$, we label the Green function of the disconnected system as $\mathcal{G}_{j, j^{\prime}}(E, k)$ describing electronic propagation between lines $j$ and $j^{\prime}$. To distinguish between atomic lines on the tube and on the substrate, we label the former by integers $j \leqslant 0$ and the latter by $j \geqslant 1$. It is clear that $\mathcal{G}_{j, j^{\prime}}$ vanishes if $j$ and $j^{\prime}$ refer to lines on different subsystems.

The effect of the contact on the total density of states can be calculated by summing up the corresponding change in the Green function over all possible lines, i.e., $\Sigma_{j} \Delta G_{j, j}$, where $\Delta G_{j, j}=G_{j, j}-\mathcal{G}_{j, j}$ gives the variation of the Green function evaluated at line $j$. Consistently with our notation, we define lines $j=0$ and $j=1$ as those atomic lines connecting the tube with the substrate, respectively. Therefore, the matrix elements of $\Delta G_{j, j}$ are

$$
\Delta G_{j, j}=\mathcal{G}_{j, 0} t\left(1-\mathcal{G}_{1,1} t^{\dagger} \mathcal{G}_{0,0} t\right)^{-1} \mathcal{G}_{1,1} t^{\dagger} \mathcal{G}_{0, j}
$$

and

$$
\Delta G_{j, j}=\mathcal{G}_{j, 1} t^{\dagger}\left(1-\mathcal{G}_{0,0} t \mathcal{G}_{1,1} t^{\dagger}\right)^{-1} \mathcal{G}_{0,0} t \mathcal{G}_{1, j},
$$

for lines on the tube $(j \leqslant 0)$ and on the substrate $(j \geqslant 0)$, respectively. In the equations above, we have introduced the parameter $t$ describing the coupling between the tube and the substrate. This quantity plays the role of a tight-binding-like energy-independent electronic hopping between the relevant overlapping orbitals on either side. The Green functions above are energy- and $k$-dependent matrices, whose indices may refer to orbital and spin degrees of freedom.

We define $\Delta \rho(E)=\rho(E)-\rho_{0}(E)$ as the density of states change between the disconnected $\left(\rho_{0}\right)$ and the sidecontacted system $(\rho)$. According to Eq. (1), the variation of the total density of states is given by

$$
\Delta \rho(E)=\left(-\frac{1}{\pi}\right) \operatorname{Im} \operatorname{Tr} \sum_{k} \sum_{j} \Delta G_{j, j}(E, k),
$$

where the trace accounts for possible internal degrees of freedom such as spin and orbital indices. By combining the cyclic property of the trace with the definition of Green functions, we can write $\Delta \rho(E)$ as

$$
\begin{aligned}
\Delta \rho(E)= & -\left(\frac{1}{\pi}\right) \operatorname{Im} \operatorname{Tr} \sum_{k}\left(1-\mathcal{G}_{0,0} t \mathcal{G}_{1,1} t^{\dagger}\right)^{-1} \\
& \times\left[\frac{d \mathcal{G}_{0,0}}{d E} t \mathcal{G}_{1,1} t^{\dagger}+\mathcal{G}_{0,0} t \frac{d \mathcal{G}_{1,1}}{d E} t^{\dagger}\right],
\end{aligned}
$$

or in a further simplified form

$$
\Delta \rho(E)=-\left(\frac{1}{\pi}\right) \operatorname{Im} \sum_{k} \frac{d}{d E} \ln \operatorname{det}\left(1-\mathcal{G}_{0,0} t \mathcal{G}_{1,1} t^{\dagger}\right) .
$$

Equation (6) writes $\Delta \rho(E)$ in terms of the Green function matrix elements for the disconnected system $(\mathcal{G})$ plus the coupling parameters $t$ and $t^{\dagger}$ between the tube and substrate. More specifically, it only depends on the diagonal elements $\mathcal{G}_{0,0}$ and $\mathcal{G}_{1,1}$, namely, those where the connection takes place. Equation (6) is therefore a convenient expression to calculate the effect of the coupling since it provides the required change in the density of states without the need of evaluating the electronic structure for the connected and disconnected systems separately. This is equivalent to the Lloyd's formula ${ }^{9}$ describing the variation of density of states due to a diagonal perturbation, a very useful method to treat substitutional impurities. This method has also been used in the study of magnetic coupling between impurities in metallic systems. ${ }^{10}$ The fundamental difference in the case presented here is that the perturbation has an off-diagonal form representing the contact between the two structures. It is 
worth highlighting that the expression above involves no approximation and is exact for arbitrary values of the parameters $t$ and $t^{\dagger}$

From the variation in the total density of states, we can derive the change in the number of electrons $\Delta N$ (at zero temperature). This is obtained by integrating $\Delta \rho$ up to the Fermi level $E_{F}$, i.e.,

$$
\Delta N\left(E_{F}\right)=-\left(\frac{1}{\pi}\right) \sum_{k} \operatorname{Im} \ln \operatorname{det}\left[1-\mathcal{G}_{0,0}\left(E_{F}\right) t \mathcal{G}_{1,1}\left(E_{F}\right) t^{\dagger}\right]
$$

In the equation above, the relevant matrix elements are evaluated at the Fermi level. Since $\Delta \rho(E)$ is the variation of the total density of states, its integral gives the change of number of particles in the closed system. This is of course a conserved quantity and the equation $\Delta N\left(E_{F}\right)=0$ allows us to calculate the value of the Fermi level.

Another quantity derivable from the change in the density of states is the effect of the contact on the total electronic energy. This is a fundamental quantity whose value determines whether or not the perturbation in the electronic structure is energetically favorable. It is defined as

$$
\Delta E=\int_{-\infty}^{E_{F}} d E E \Delta \rho(E)
$$

From the expression for $\Delta \rho(E)$ in Eq. (6) we have that

$$
\Delta E=\left(\frac{1}{\pi}\right) \sum_{k} \int_{-\infty}^{E_{F}} d E \operatorname{Im} \ln \operatorname{det}\left(1-\mathcal{G}_{0,0} t \mathcal{G}_{1,1} t^{\dagger}\right) .
$$

If $\Delta E<0$, the changes in the electronic structure predicted by Eqs. (6), (7), and (9) are possible when the energy gain is sufficient to overcome the energy costs involved in the transition.

The changes $\Delta \rho, \Delta N$, and $\Delta E$ describe the effect of the contact on the density of states, number of particles, and total energy of the entire system, i.e., the tube and the substrate. In order to investigate possible contact-induced spin polarizations, one must look at similar changes on the separate parts. In other words, instead of evaluating the total density of states summed over all possible sites in the structure, we must distinguish between the changes in the tube and in the substrate. Bearing in mind that global charge neutrality is imposed by Eq. (7), any modification in the total number of particles on the tube must be compensated by the corresponding change on the substrate. Therefore, to calculate the charge transfer between the tube and substrate it is sufficient to evaluate the variation of number of particles on either part. We choose to focus on the tube and calculate the change of density of states summed over all atomic lines of the tube. Analogously to the derivation presented above, the change in the density of states $\left(\rho_{t}\right)$ on the tube is given by

$$
\Delta \rho_{t}(E)=\left(\frac{1}{\pi}\right) \operatorname{Im} \operatorname{Tr} \sum_{k}\left(1-\mathcal{G}_{1,1} t^{\dagger} \mathcal{G}_{0,0} t\right)^{-1} \frac{d \mathcal{G}_{0,0}}{d E} t \mathcal{G}_{1,1} t^{\dagger}
$$

The charge transfer to the tube $\left(\Delta N_{t}\right)$ is the integral of the above expression and is written as

$$
\begin{aligned}
\Delta N_{t}(E)= & \left(\frac{1}{\pi}\right) \operatorname{Im} \operatorname{Tr} \sum_{k} \int_{-\infty}^{E_{F}} d E\left(1-\mathcal{G}_{1,1} t^{\dagger} \mathcal{G}_{0,0} t\right)^{-1} \\
& \times \frac{d \mathcal{G}_{0,0}}{d E} t \mathcal{G}_{1,1} t^{\dagger} .
\end{aligned}
$$

Equations (9) and (11) are the fundamental results of this work. The first tells us whether or not the charge transfer is energetically favorable, and the second the amount of charge exchanged between the tube and the substrate. These form a closed system of equations written in terms of the Green functions of the disconnected system and the coupling parameters. It is important to stress that although the complexity involved in evaluating the expressions for $\Delta N_{t}$ and $\Delta E$ depends on the choice of the Hamiltonian used to describe the electronic structure of the system, the validity of Eqs. (9) and (11) does not. This means that our expressions can be equally used with simple model Hamiltonians or with a full realistic description of the electronic structure.

Equation (10) for $\Delta \rho_{t}$ is not as concise as its counterpart Eq. (6) but it can be further simplified by expanding it to second order in $t$. This approximation is valid in the limit of weak coupling, which is satisfied in the case of carbon nanotubes sitting on top of transition metals. In fact, recent density-functional-theory calculations of graphite on a [001] cobalt surface suggest a value for the coupling parameter of $t=W / 30$, where $W$ is the width of the graphite $\pi$ band. ${ }^{11}$ This value has been estimated using the general tight-binding scaling law ${ }^{12}$ for the $p d \sigma$ hopping parameter between the $\mathrm{C} p_{z}$ and the Co $d_{z^{2}}$ orbitals. Furthermore, when the sum over $k$ is eliminated and $\Delta \rho_{t}(E)$ is integrated up to the Fermi level, $\Delta N_{t}\left(E_{F}\right)$ becomes

$$
\Delta N_{t}\left(E_{F}\right)=\operatorname{Tr} \int_{-\infty}^{E_{F}} d E\left[\rho_{0}(E) \frac{d V_{1}(E)}{d E}+\rho_{1}(E) \frac{d V_{0}(E)}{d E}\right],
$$

where $\rho_{0}(E)$ and $\rho_{1}(E)$ are the density of states on the tube and on the substrate, respectively. $V_{m}(E)=t \operatorname{Re}\left[\mathcal{G}_{m, m}\right] t^{\dagger}(m$ $=0,1)$ plays the role of an energy-dependent electronic potential. $V_{1}(E)$ is the potential felt by the tube due to the substrate and $V_{0}(E)$ is the analogous potential felt by the substrate and produced by the tube. It is worth noting that the potentials $V_{0}(E)$ and $V_{1}(E)$ depend on the real part of the Green functions $\mathcal{G}_{0,0}$ and $\mathcal{G}_{1,1}$, respectively. These two quantities are directly obtainable from electronic structure calculations for the disconnected system. A similar second-order expansion of Eq. (9) also simplifies the formula for the energy change $\Delta E$, which now reads as

$$
\Delta E=\operatorname{Tr} \int_{-\infty}^{E_{F}} d E\left[\rho_{0}(E) V_{1}(E)+\rho_{1}(E) V_{0}(E)\right] .
$$

Although Eqs. (12) and (13) represent a more concise version of their respective counterparts, Eqs. (11) and (9), they are still in integral forms. These can be further simplified by replacing $V_{m}(E)$ with its mean value $\left\langle V_{m}(E)\right\rangle$. This approximation gives rise to the following two expressions: 


$$
\begin{aligned}
\Delta N_{t}\left(E_{F}\right)= & \operatorname{Tr}\left\{\rho_{0}\left(E_{F}\right)\left[V_{1}\left(E_{F}\right)-\left\langle V_{1}\right\rangle\right]\right. \\
& \left.+\rho_{1}\left(E_{F}\right)\left[V_{0}\left(E_{F}\right)-\left\langle V_{0}\right\rangle\right]\right\}
\end{aligned}
$$

and

$$
\Delta E=\operatorname{Tr}\left\{N_{0}\left(E_{F}\right)\left\langle V_{1}\right\rangle+N_{1}\left(E_{F}\right)\left\langle V_{0}\right\rangle\right\} .
$$

In this form, the expressions for the charge transfer and the respective energy gain are written in terms of the density of states for both the tube and the substrate, and the potentials $V_{0}\left(E_{F}\right)$ and $V_{1}\left(E_{F}\right)$, all evaluated at the Fermi level. They also depend on the total number of electrons $N_{0}$ and $N_{1}$.

In calculating the average $\left\langle V_{0}\right\rangle\left(\left\langle V_{1}\right\rangle\right)$, the integration limits are not in the range $\left[-\infty, E_{F}\right]$ as in Eqs. (12) and (13) but start from the bottom of the electronic band $\rho_{1}\left(\rho_{0}\right)$. The upper integration limit is common to both cases and is given by the Fermi level $E_{F}$. It is clear from Eq. (14) that the sign of $\Delta N_{t}\left(E_{F}\right)$ is fully determined by the potentials $V_{0}$ and $V_{1}$. The side-contacted nanotube will then be electron- (hole-) doped for positive (negative) values of $\Delta N_{t}\left(E_{F}\right)$. This means that the balance between $\left\langle V_{m}\right\rangle$ and $V_{m}\left(E_{F}\right)$ determines the type of charge transfer between the structures. We have checked the results obtained by the mean-field equations against those predicted by Eqs. (11) and (9), and we find both a qualitative agreement and values of the same order of magnitude.

\section{MAGNETIC SUBSTRATES}

The expressions presented in the preceding section display no explicit dependence of the electronic structure on the spin degree of freedom. However, when a magnetic substrate is considered, the spin symmetry is broken. In this case the expressions derived in the preceding section are still valid since an explicit spin dependence can be added to both the Green functions and the coupling parameter, without loss of generality. Although general noncollinear spin Hamiltonians can be considered, we restrict our analysis to collinear spin in the two-spin fluid model. Within this model all the quantities are diagonal in the spin subspace and the only variation with the spin-degenerate case is that the expressions for the charge transfer and the energy gain are different for the two spin subbands. Therefore any induced magnetization $M$ on the tube results from the spin imbalance of the charge transfer, $M=\left(\Delta N_{t}^{\uparrow}-\Delta N_{t}^{\downarrow}\right) \mu_{B}$, where $\Delta N_{t}^{\sigma}$ is the charge transfer for a spin $\sigma$ and $\mu_{B}$ is the Bohr magneton. When the substrate is magnetic the charge transfer for the majority-spin subband is different from that of the minority, leading to a net induced magnetic moment on the tube.

It is worth recalling that Eqs. (12) and (13) are complementary and that charge will be transferred only if the corresponding energy gain is sufficient to outweigh the energy costs. In the two-spin fluid model we have to calculate the energetics of the charge transfer process for each spin direction. Only when the energy gain is favorable for both spins does the quantity $\left(\Delta N_{t}^{\uparrow}-\Delta N_{t}^{\downarrow}\right) \mu_{B}$ describe the induced moment. In other words, if the energy gain for one spin direction is not sufficient to surpass the energy costs, the corresponding charge transfer will not take place and the difference $M=\left(\Delta N_{t}^{\uparrow}-\Delta N_{t}^{\downarrow}\right) \mu_{B}$ will be meaningless. Assuming that both transitions are energetically favorable we can make use of Eq. (12) to write the induced moment $M$ as

$$
\begin{aligned}
M= & \mu_{B} \operatorname{Tr} \int_{-\infty}^{E_{F}} d E\left\{\rho_{0}(E) \frac{d}{d E}\left[V_{1}^{\uparrow}(E)-V_{1}^{\downarrow}(E)\right]\right. \\
& \left.+\left[\rho_{1}^{\uparrow}(E)-\rho_{1}^{\downarrow}(E)\right] \frac{d V_{0}(E)}{d E}\right\},
\end{aligned}
$$

where the spin polarization $\sigma$ is now explicitly included in the quantities describing the substrate. Bearing in mind that the spin bands are split by the exchange integral $\Delta$ and neglecting possible hybridization effects, we can approximately correlate the majority- and minority-spin bands by $V_{1}^{\downarrow}(E)$ $=V_{1}^{\uparrow}(E-\Delta)$ and $\rho_{1}^{\downarrow}(E)=\rho_{1}^{\uparrow}(E-\Delta)$. A further simplification can be made by expanding the substrate quantities in powers of $\Delta$. In this case, the induced magnetization becomes

$M=-\mu_{B} \Delta \operatorname{Tr} \int_{-\infty}^{E_{F}} d E\left\{\rho_{0}(E) \frac{d^{2} V_{1}^{\uparrow}(E)}{d E^{2}}+\frac{d \rho_{1}^{\uparrow}(E)}{d E} \frac{d V_{0}(E)}{d E}\right\}$

Both equations (16) and (17) give the induced magnetic moment in terms of quantities that are directly obtainable from electronic structure calculations and provide valuable expressions to determine the contact-induced spin polarizations. Whereas the latter is valid for magnetic substrates whose spin bands are not significantly split, the former gives a general expression for the induced moment with no limitations about the electronic structure parameters.

In order to test whether or not the magnetic contact can induce spin polarization on the tube we must determine the order of magnitude of the charge transfer for a given spin band. This can be done within a simplified model that contains the fundamental features of the electronic structure of both the nanotube and the substrate. The single-band tightbinding model is known to reproduce well the band structure of both graphite and nanotubes of somewhat large diameters. The electronic hopping within the tube is described by the parameter $\gamma=2.5 \mathrm{eV}$ and is hereafter used as our energy unit. Likewise, the $d$ band of magnetic transition metals can be described within the same model by an appropriate choice of band width and total number of electrons. Figure 1 shows the density of states $\rho_{0}$ and $\rho_{1}$ as well as the potentials $V_{0}$ and $V_{1}$ for a typical case. We have chosen an armchair nanotube with $N=12$ atoms per ring. The substrate is modeled by a semi-infinite cubic slab whose electronic structure parameters lead to a band that is 5-eV wide (typical of transition metals) and that is centered at an arbitrary position $\epsilon_{1}$ $=0.75 \gamma$. The Fermi level is fixed at $E_{F}=0$ and is represented in the figure by a vertical line. For this choice of parameters, the calculated results are a charge transfer of $\Delta N_{t}=-2 \times 10^{2}$ electrons $/ \mu \mathrm{m}$ with the respective energy gain of $\Delta E=-6.5 \times 10^{-2} \mathrm{eV}$ per unit cell. It is worth recalling that the present calculations are for zero temperature, although the finite-temperature regime can in principle be accounted for by including the Fermi functions in the inte- 


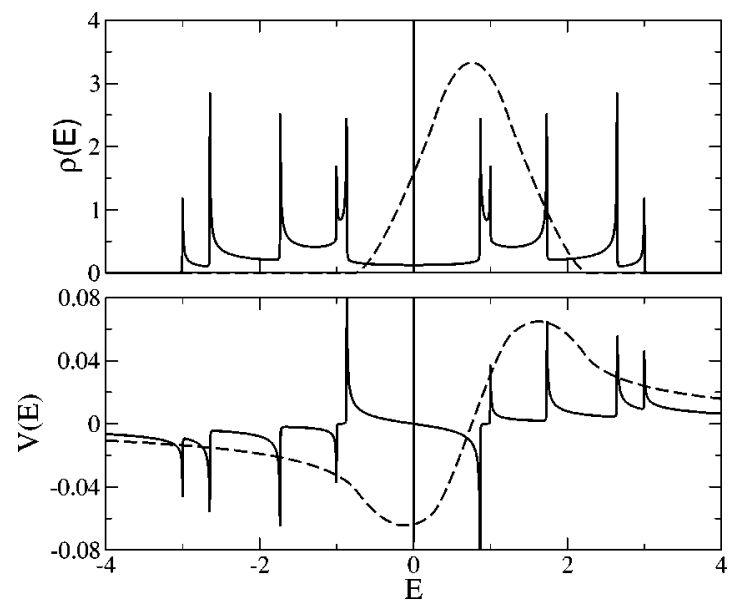

FIG. 1. Density of states $\rho_{0}$ and $\rho_{1}$ and potentials $V_{0}$ and $V_{1}$ for a typical case: a $(3,3)$ carbon nanotube (solid line) attached to a semi-infinite cubic slab (dashed line). The electronic structure parameters for the slab lead to a band that is $5 \mathrm{eV}$ wide and centered at $\epsilon_{1}=0.75 \gamma$. The Fermi level is fixed at $E_{F}=0$ (vertical line).

grands of the expressions above. The negative sign of $\Delta N_{t}$ indicates that for this particular band alignment, electrons flow from the nanotube onto the substrate.

The specific values of $\Delta N_{t}$ and $\Delta E$ depend on the particular alignment of the electronic bands of the tube and the substrate. We investigate different possibilities by changing the on-site potential $\epsilon_{1}$ of the substrate atoms. This corresponds to shifting the band center along the nanotube energy spectrum. In Fig. 2 we show the changes $\Delta N_{t}$ and $\Delta E$ as a function of $\epsilon_{1}$ for two different tube diameters. Since the Fermi energy is kept at $E_{F}=0$, a shift in the electronic band of the substrate also affects the total number of electrons $N_{1}$ on the substrate. The figure shows that the charge transfer $\Delta N_{t}$ can change sign, indicating that the tube can be doped either with electrons or with holes. However, despite differ-
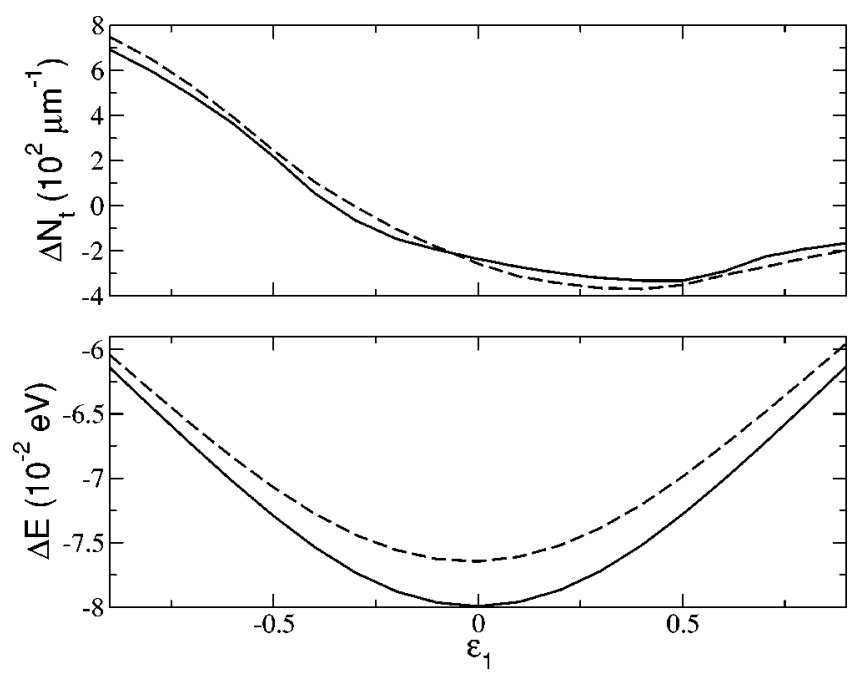

FIG. 2. Charge transfer $\left(\Delta N_{t}\right)$ and the respective energy gain per unit cell $(\Delta E)$ for different band alignments. The parameter $\epsilon_{1}$ corresponds to the center of the substrate band. Solid and dashed lines refer to $(3,3)$ and $(8,8)$ armchair nanotubes, respectively. ences in sign, the magnitude of the charge transfer $\left|\Delta N_{t}\right|$ does not change substantially and reaches values up to 8 $\times 10^{2}$ electrons $/ \mu \mathrm{m}$. The fact that the charge transfer between the tube and the substrate can change sign depending on the band alignment, has important consequences on the induced magnetic moment. In fact, if the band splitting of the substrate is such that the charge transfer for the majority-spin subband has opposite sign to that of the minority-spin band, the spin balance on the nanotube is not only broken but maximized. In this case electrons of opposite spins flow in opposite directions (for instance, majority spins will flow from the substrate into the tube, and minority from the tube into the substrate). The picture shows that this is the case when the Fermi level lies close to opposite edges of the ferromagnetic spin bands. Half metals seem to satisfy this requirement and therefore are potential candidates for inducing large spin imbalance in nanotubes. In other words, magnetic substrates made of half metals are predicted to be the best materials to induce a magnetic moment in a nanotube. Considering the results of Fig. 2 as a reference, we estimate that the maximum value of induced magnetization is $M$ $=10^{-1} \mu_{B}$ per unit cell, a magnitude that is experimentally detectable. The stability of this induced moment can also be estimated by the energy gain $\Delta E$, which is in the order of $10^{-1} \mathrm{eV}$, as shown in Fig. 2. Induced moments in tubes of increasingly large diameters are less stable and $\Delta E$ must saturate toward the value associated with a nanotube deposited on a graphite substrate. Furthermore, we note that the lattice relaxation ${ }^{13}$ induced by the charge transfer as well as the charging energy due to the low capacitance of carbon nanotubes ${ }^{14}$ must be considered when calculating the total energy costs of the electronic transition. Although these two factors are influential on whether the transfer process becomes energetically favorable, they are at least one order of magnitude below the energy gain obtained by the change in the electronic structure. In fact, the typical value for the quantum capacitance $^{15}$ (per unit cell) of a nanotube is $C$ $=0.35 \mathrm{e} / \mathrm{V}$. For the parameters used in Fig. 1, this leads to a charging energy $E_{c}=0.0025 \mathrm{eV}$, which is still substantially smaller that the energy gain of $\Delta E=0.065 \mathrm{eV}$ calculated here.

Finally, we wish to briefly comment on the similarities between our contact-induced spin polarization effect and the problem of spin-injection from metallic systems. Whereas the latter is a nonequilibrium transport effect, the former is the result of charge and spin rearrangement toward the equilibrium configuration between the magnetic and nonmagnetic materials. In other words, while in the spin-injection problem the electrons must travel long distances to be probed by a detector, this is not the case for the contact-induced spin-polarization. The two phenomena can indeed be addressed by a common formalism but a complete comparison would require a reformulation of our method in terms of transport quantities, which is beyond the scope of the present paper.

\section{CONCLUSIONS}

In summary, we have presented a model that describes the charge transfer of a carbon nanotube in contact to a substrate. 
Closed-form expressions in terms of Green functions give the charge transfer and the respective energy gain associated with the transition. We have subsequently shown that when the substrate is magnetic the spin imbalance of the surface may lead to an induced spin polarization in the nanotube. Within a simple model that reproduces the basic features of the electronic structures of both nanotubes and transition metal surfaces, we were able to estimate the magnitude and the stability of the induced moment. This indicates that a measurable magnetic moment can be induced in carbon nanotubes when contacted to magnetic substrates. Finally, we have demonstrated that half metals are the best candidates for inducing a sizable magnetic moment in carbon nanotubes.

\section{ACKNOWLEDGMENTS}

The authors are grateful to C. J. Lambert, J. M. D. Coey, and R. B. Muniz for helpful discussions. S.S. thanks Enterprise Ireland (Grant No. EI-SC/2002-10) for the financial support.
${ }^{1}$ S.A. Wolf, D.D. Awschalom, R.A. Buhrman, J.M. Daughton, S. von Molnar, M.L. Roukes, A.Y. Chtchelkanova, and D.M. Treger, Science 294, 1488 (2001).

${ }^{2}$ M.N. Baibich, J.M. Broto, A. Fert, F. Nguyen Van Dau, F. Petroff, P. Etienne, G. Creuzet, A. Friederich, and J. Chazelas, Phys. Rev. Lett. 61, 2472 (1988).

${ }^{3}$ C. Joachim, J.K. Gimzewski, and A. Aviram, Nature (London) 408, 541 (2000).

${ }^{4}$ S.J. Tans, A.R.M. Verschueren, and C. Dekker, Nature (London) 393, 49 (1998).

${ }^{5}$ J. Chen, M.A. Reed, A.M. Rawlett, and J.M. Tour, Science 286, 1550 (1999).

${ }^{6}$ Z. Yao, H.W.Ch. Postma, L. Balents, and C. Dekker, Nature (London) 402, 273 (1999).
${ }^{7}$ K. Tsukagoshi, B.W. Alphenaar, and H. Ago, Nature (London) 401, 572 (1999).

${ }^{8}$ J.M.D. Coey, M. Venkatesan, C.B. Fitzgerald, A.P. Douvalis, and I.S. Sanders, Nature (London) 420, 156 (2002).

${ }^{9}$ P. Lloyd, Proc. Phys. Soc. 90, 207 (1967).

${ }^{10}$ B.C. Lee and Y.C. Chang, Phys. Rev. B 62, 3888 (2000).

${ }^{11}$ K. Goto, N. A. Spaldin, and S. Sanvito (unpublished).

${ }^{12}$ W.A. Harrison, Electronic Structure and the Properties of Solids (Dover, 1980).

${ }^{13}$ M. Verissimo-Alves, B. Koiller, H. Chacham, and R.B. Capaz, Phys. Rev. B 67, 161401 (2003).

${ }^{14}$ P. Pomorski, C. Roland, H. Guo, and J. Wang, Phys. Rev. B 67, 161404 (2003).

${ }^{15}$ K.A. Bulashevich and S.V. Rotkin, JETP Lett. 75, 205 (2002). 